\title{
Case of idiopathic granulomatous mastitis: Recognition of a new management paradigm
}

\author{
Laura M Ochoa-Frongia ${ }^{1}$, David R Anderson ${ }^{2}$, Lakeeya D Tucker ${ }^{3}$ and Harvey L Bumpers ${ }^{1 *}$ \\ ${ }^{1}$ Departments of Surgery, Michigan State University, CHM, East Lansing, MI, USA \\ ${ }^{2}$ Departments of Radiology, Michigan State University, CHM, East Lansing, MI, USA \\ ${ }^{3}$ Alliance Obstetrics and Gynaecology, East Lansing, MI, USA
}

\begin{abstract}
Granulomatous mastitis is a benign breast disease where the difficulty in diagnosis is due to its sporadic nature, rarity, lack of awareness amongst providers, and variable features that often can be mistaken for infectious or malignant diseases. The diagnosis is made with core needle biopsy showing giant multinucleated cells, epithelioid histiocytes, lymphocytic infiltration and absence of bacteria or malignant cellular transformation. Once diagnosis is made, initial treatment is a course of high-dose steroids; the patient is monitored in clinic for resolution. After the inflammatory process regresses, the residual atypical tissue is surgically excised with clear margins. Prompt diagnosis and proper treatment results in long term resolution.
\end{abstract}

\section{Introduction}

Despite over forty years since the first major publication [1] identifying idiopathic granulomatous mastitis (IGM), this benign breast disease has remained enigmatic. Sporadic case reports [2] have been published in journals internationally since then; only several case series of over ten patients or multicenter retrospective studies have been performed. Therefore, the pre-diagnosis patient experience remains almost identical to that from a generation ago that occurred outside enters with expertise in breast disease. Without IGM on the early differential, diagnosis follows a perplexing presentation and protracted course of ruling out of other conditions. Even today, with unfortunate regularity, a surprising proportion of patients endure multiple iterations of misdiagnosis and therapeutic failure before arriving at the correct diagnosis [3]. Though its rarity has contributed to the challenge of identifying the etiology of this disease, some features are common: female, childbearing age, history of previous viral or autoimmune process, certain non-white ethnicities such as Asians, Hispanics and those of Middle Eastern descent [4,5]. With each new case recorded, more data is added to fill in the narrowing gaps in understanding.

\section{Patient case}

The patient is a 38 year woman referred urgently by her gynaecologist with a left breast mass that had rapidly filled the upper inner quadrant (UIQ) with concerns for inflammatory breast cancer. The patient complained of pain and mild redness of the skin over the anterior breast. She denied trauma, fever, chills, nipple discharge, or skin ulceration. The breast exam revealed obvious breast asymmetry due to a $15 \times 15 \mathrm{~cm}$ mass extending from the UIQ to an area below the nipple-areola complex. It was firm, irregular, with mild skin edema, and mobile relative to the chest wall (Figure 1). There was no axillary adenopathy. Mammography (Figure 2), ultrasound, and biopsies were obtained. Pathology reported acute and chronic mastitis with granulomatous reaction and negative for carcinoma.

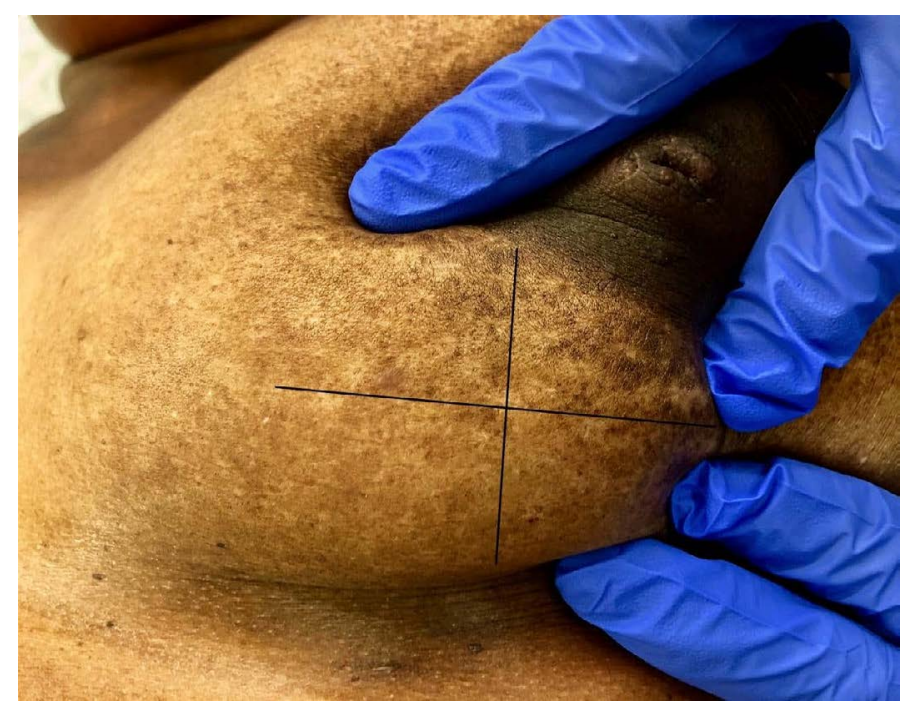

Figure 1. Palpable left breast mass and size is $15 \mathrm{~cm} \times 15 \mathrm{~cm}$

\section{Treatment}

The patient received four weeks of high-dose steroids (methylprednisolone $0.5 \mathrm{mg} / \mathrm{kg}$ per day in divided doses) followed by taper. At re-evaluation two weeks later, the mass had become a subtle density and measured $3 \mathrm{~cm}$ in maximum dimension. Excision of residual diseased tissue was then undertaken to prevent recurrence.

${ }^{\star}$ Correspondence to: Harvey L. Bumpers, Department of Surgery Michigan State University, CHM 4660 S Hagadorn Rd, Suite 600 East Lansing, MI 48823, USA, Tel: 86-21-64931928; E-mail: Harvey.bumpers@hc.msu.edu

Key words: granulomatous mastitis, mastitis, granulomatous breast

Received: May 12, 2019; Accepted: May 22, 2019; Published: May 24, 2019 


\section{Results}

A four-week course of high-dose steroids was completed with nearcomplete resolution of the palpable lesion and clinical symptoms. The residual lesion was excised completely (Figure 3 ) with negative margins. Histopathology (Figure 4) was consistent with the previous biopsy and confirmed diagnosis of IGM. The patient remains asymptomatic 10 months after surgery.

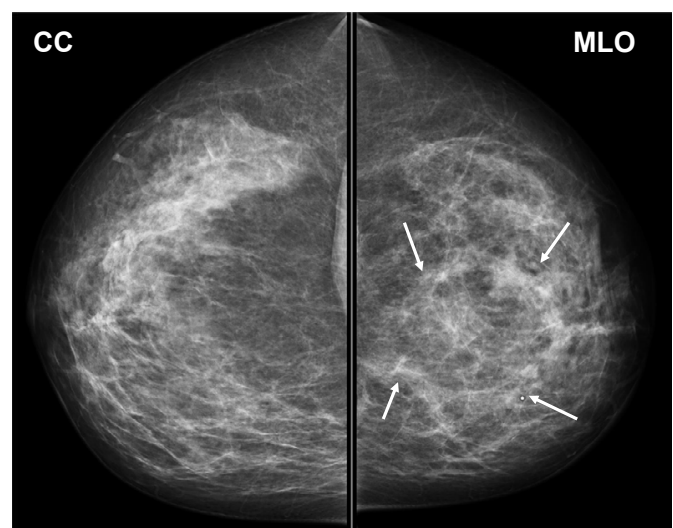

Figure 2. Pretreatment Diagnostic mammogram. Arrows denote the margins of the mass

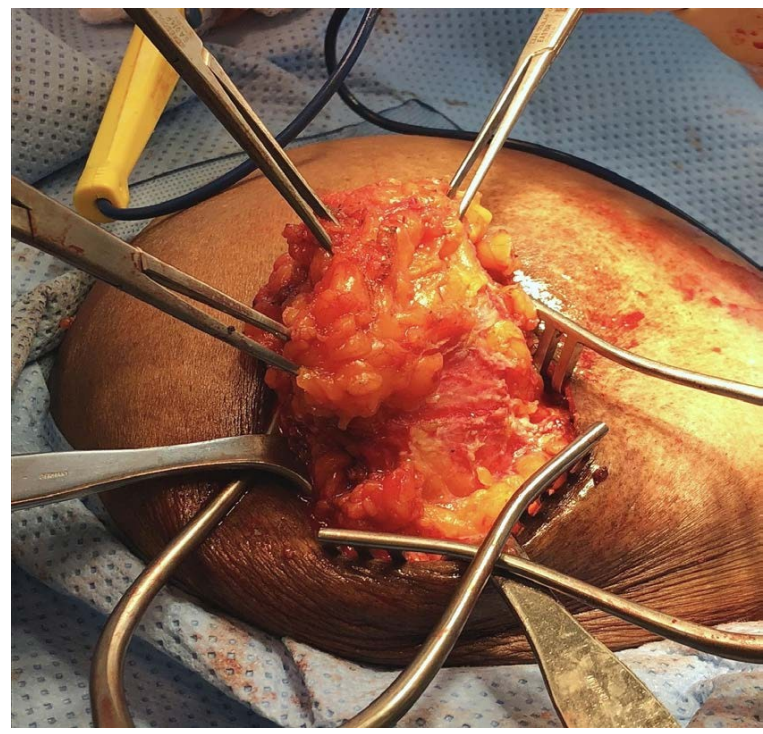

Figure 3. Excision of residual granuloma tissue

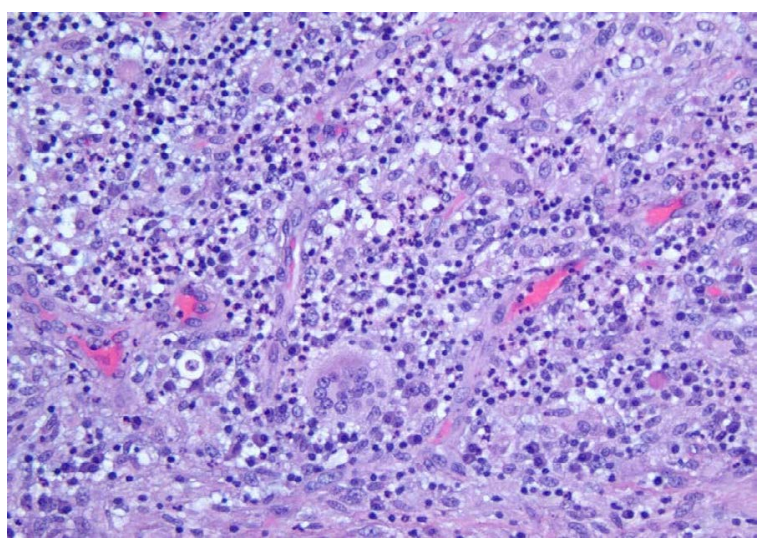

Figure 4. Surgical pathology: Multinucleated giant cells, epithelioid histiocytes, and lymphocytic infiltrate forming non-caseating granulomas around ducts and lobules.

\section{Discussion}

Granulomatous mastitis is most commonly mistaken clinically and radiologically as a bacterial infection or an inflammatory breast cancer. There is no widely described autoimmune condition with similar features. Typically, arrival at the diagnosis occurs following several weeks to months of anxiety and significant pain if the process remains untreated for that duration. Delay in diagnosis is due to multiple factors, beginning with the rarity and under-reporting of the disease [2]. Clinically, features of the physical exam and mammogram can closely resemble inflammatory breast cancer, similarly, the characteristic discharge is often mistaken as the purulence of a bacterial process and treatment is started presumptively without core needle biopsy [5]. In less fortunate cases, the diagnosis comes after failures of protracted courses of unnecessary antibiotics or chemotherapy.

At worst, patients undergo surgical intervention, only to have the disease progress with profound exacerbation and spread to other sites in the same or even contralateral breast. Clinical acumen is important, as early recognition and treatment lead to a better response [6]. Though noncancerous, the term "benign" refers to histology, not patient impact. Without an efficient diagnosis and therapy, the duration of symptoms and treatment can last longer than that experienced for localized breast cancer. This painful and disfiguring disease requires ongoing investigation to end the nearly 50-year-long poor understanding of pathophysiology and lack of consensus on a treatment regimen. Recent increase in publications and awareness are noted, and new data shows stronger connection with autoimmune processes.

\section{Conclusion}

Prompt diagnosis and initiation of treatment is crucial to the clinical response and long-term resolution of IGM. High dose steroid is the initial treatment of choice for granulomatous mastitis. Though surgery is often necessary to remove residual disease, it is increasingly utilized after completion of first-line medical therapy. Primary surgical intervention is no longer the mainstay of treatment.

\section{References}

1. Freeman CM, Xia BT, Wilson GC, Lewis JD, Khan S, et al. (2017) Idiopathic granulomatous mastitis: a diagnostic and therapeutic challenge. Am J Surg 214: 701706. [Crossref]

2. Kessler E, Wolloch Y (1972) Granulomatous Mastitis: A Lesion Clinically Simulating Carcinoma. Am J Clin Pathol 58: 642-646. [Crossref]

3. Baslaim MM, Khayat HA, Al-Amoudi SA (2007) Idiopathic granulomatous mastitis: a heterogeneous disease with variable clinical presentation. World J Surg 31: 1677-1681. [Crossref]

4. Sheybani F, Naderi HR, Gharib M, Sarvghad M, Mirfeizi Z (2016) Idiopathic granulomatous mastitis: Long-discussed but yet-to-be-known. Autoimmunity 49: 236239. [Crossref]

5. Pluguez-Turull CW, Nanyes JE, Quintero CJ, Alizai H, Mais DD, et al. (2018) Idiopathic Granulomatous Mastitis: Manifestations at Multimodality Imaging and Pitfalls. Radiographics 38: 330-356. [Crossref]

6. Bashir MU, Ramcharan A, Alothman S, Beaugris S, Khan SA, et al. (2017) The enigma of granulomatous mastitis: a series. Breast Dis 37: 17-20. [Crossref]

Copyright: (C2019 Ochoa-Frongia LM. This is an open-access article distributed under the terms of the Creative Commons Attribution License, which permits unrestricted use, distribution, and reproduction in any medium, provided the original author and source are credited. 